\title{
Slovenian Discrete and Applied Mathematics Society Joins the EMS
}

Klavdija Kutnar and Tomaž Pisanski (both University of Primorska, Koper, Slovenia)

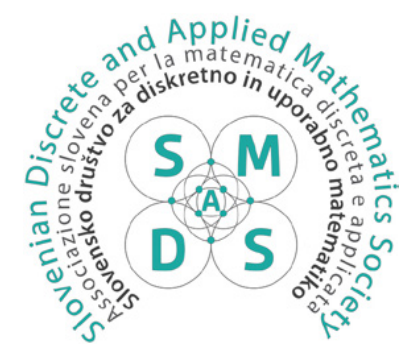

Slovenian Discrete and Applied Mathematics Society, founded in December 2016.
At the EMS council meeting in Prague, 23-24 June 2018, the young Slovenian Discrete and Applied Mathematics Society (SDAMS) was accepted as a full member of the EMS. There are now 56 full member societies from 44 countries. Slovenia joined the group of countries that have more than one full member society: Spain (4), Italy (3), UK (3), France (3), Germany (2) and Russia (2). It is the first European country with a member society that has the word discrete in its title and the first society from a country behind the former Iron Curtain that has the name applied in its title.

It seems to be a bit unusual for a country of two million inhabitants to have two mathematical societies. However, we want to show that this is a natural step in the development of mathematics in Slovenia.

Firstly, we present a brief overview of the historical development of Slovenian mathematics. 


\section{Historical development of mathematics in Slovenia Habsburg rule until 1918}

For most of its history, Slovenia was under Habsburg rule, which ended in 1918 after World War I. Slovenian mathematicians who were born in Austria, or later in the Austro-Hungarian empire, include Herman de Carinthia, also known as Herman Dalmata, who translated Euclid's Elements from Arabic to Latin, Andrej Perlach, who became a rector of the University of Vienna and who taught diverse subjects such as mathematics and medicine, Jurij Vega, known for his logarithm tables and calculations of digits of $\pi$, Franc Močnik, one of the most prolific writers of high school textbooks in the Austrian Empire (and translated into 12 languages), Franc Hočevar, the first Slovenian mathematician who published papers in the modern sense, Josip Plemelj, known for instance for the Sokhotski-Plemelj theorem from complex analysis, Ivo Lah, known for Lah numbers in combinatorics, and Ivan Vidav of the Vidav-Palmer theorem from functional analysis.

\section{Kingdom of Yugoslavia, from 1918 until 1945}

In 1919, the University of Ljubljana was founded and Plemelj became its first rector. He focused on producing a high quality curriculum, essentially covering algebra and number theory, differential equations and analytic functions. Unfortunately, he only published the corresponding three textbooks at the end of his career after World War II. Nearly all students of mathematics at that time became high school teachers of mathematics, with two exceptions. His first PhD student, Anton Vakselj (PhD in 1924), became a professor of mathematics at the Technical Faculty of the University of Ljubljana while his second PhD student Ivan Vidav (PhD in 1941) became his successor.

\section{Socialist Federal Yugoslavia, from 1945 until 1991}

In 1949, the Society of Mathematicians, Physicists and Astronomers of Slovenia was founded. At that time, less than 10 Slovenian mathematicians were involved in research in mathematics, with Plemelj and Vidav being the leading mathematicians focusing on various aspects of mathematical analysis. Although Plemelj only retired in 1957, it was Vidav who created the mathematical school in Slovenia. Between 1955 and 1985, he had 17 $\mathrm{PhD}$ students and currently has 105 academic descendants. ${ }^{1}$ However, in the 1970 s, a number of important things happened.

In 1960, the Institute of Mathematics, Physics and Mechanics was founded in Ljubljana. In the following years, most active mathematicians in Slovenia became partially employed there in order to conduct research in mathematics. This model of separating teaching at the university from research at the institute was not uncommon in Eastern Europe.

In 1972, the first graduate programme in mathematics started at the University of Ljubljana.

\footnotetext{
1 Mathematics Genealogy Project.
}

In 1973, the University of Maribor (the second university in Slovenia) was established.

Since 1972, over 20 Slovenian research mathematicians got their PhDs abroad or had foreign advisors and returned to Slovenia to pursue their academic careers at home, bringing new research areas and reducing the risk of inbreeding in a small community. As shown in Table 1 , seven of them have at least 10 academic descendants.

\begin{tabular}{|c|c|c|c|c|c|c|}
\hline (a) & (b) & (c) & (d) & (e) & $(f)$ & (g) \\
\hline Ivan Vidav & 1941 & 34 & Josip Plemelj & Austria & 17 & 105 \\
\hline Jože Vrabec & 1972 & 57 & James Cannon & USA & 2 & 11 \\
\hline Boštjan Vilfan & 1972 & 68 & $\begin{array}{l}\text { Albert deSilva } \\
\text { Meyer }\end{array}$ & USA & 4 & 10 \\
\hline Dragan Marušič & 1981 & 05 & $\begin{array}{l}\text { C. St.J.A. } \\
\text { Nash-Williams }\end{array}$ & UK & 7 & 14 \\
\hline Tomaž Pisanski & 1981 & 05 & Torrence Parsons & USA & 16 & 78 \\
\hline Franc Forstnerič & 1985 & 32 & Edgar Lee Stout & USA & 9 & 14 \\
\hline Franc Solina & 1987 & 68 & Ruzena Bajcsy & USA & 13 & 20 \\
\hline
\end{tabular}

Table 1. Mathematicians having international education with at least 10 academic descendants in Slovenia. The data are collected mostly from the Mathematics Genealogy Project:

(a) Mathematician,

(b) Year when $P h D$ was received,

(c) Math Subj Classification of the thesis,

(d) Advisor,

(e) Country,

(f) Number of doctoral students,

(g) Number of academic descendants.

These figures indicate that discrete mathematics in Slovenia, in particular combinatorics and graph theory, started in the last quarter of the 20th century.

\section{Independent Slovenia after 1991}

In an independent and democratic Slovenia, mathematics witnessed further development. Ease of travel has increased the number of mathematicians who study abroad. At the same time, the number of students from abroad is steadily increasing. Several accomplished mathematicians have also decided to continue their academic careers in Slovenia.

In 2003, the third public university, the University of Primorska, was established, followed by FAMNIT (the faculty where mathematics is taught) in 2006. Currently, all three public universities in Slovenia offer $\mathrm{PhD}$ programmes in mathematics.

In 2006, Slovenia very successfully hosted the 47th International Mathematical Olympiad (IMO), with participation of competitors and their team leaders totalling over 2000. It has a very efficient computer system for running mathematical competitions and it hosts the IMO homepage. The IMO's current secretary Gregor Dolinar also comes from Slovenia. Currently, high school competitions form a dominant activity of the Society of Mathematicians, Physicists and Astronomers of Slovenia.

In 2008, the first high-quality mathematical journal Ars Mathematica Contemporanea was established in Slovenia. It mainly covers discrete mathematics.

According to the Mathematics Genealogy Project, a little over $300 \mathrm{PhDs}$ in mathematical sciences were 
awarded by Slovenian universities, almost one half in the area of discrete and applied mathematics or theoretical computer science.

All three universities grant honorary PhDs. They all recognise the importance of mathematics. At the University of Ljubljana, the following mathematicians have been granted this honour: Josip Plemelj (1963), Alojz Vadnal (1981) and Ivan Vidav (1997) from Slovenia, and Ruzena Bajcsy and Dana Scott (2003) from the US. In 2018, Cheryl Praeger (Australia) received an honorary doctorate from the University of Primorska and, in the same year, Wilfried Imrich (Austria) received an honorary doctorate from the University of Maribor. They both work mainly in discrete mathematics.

Slovenia has attracted accomplished mathematicians to spend sabbaticals at its universities and several international mathematicians have decided to pursue their academic careers in Slovenia: one at the University of Maribor, three at the University of Ljubljana and three at the University of Primorska.

One of the biggest achievements of mathematics in Slovenia is winning the bid for the 8th European Congress of Mathematics, to take place in Portorož, Slovenia, in July 2020.

\section{A New Learned Society}

The Slovenian Discrete and Applied Mathematics Society (SDAMS) was founded in Koper (Slovenia) on 14 December 2016.

The aim of this society is to promote the mathematical sciences, with a special emphasis on discrete and applied mathematics. The society is research-oriented and publishes scientific literature and organises scientific meetings. In particular, the SDAMS is involved in publishing two international mathematical journals: Ars Mathematica Contemporanea (https://amc-journal.eu/) and The Art of Discrete and Applied Mathematics (https://adam-journal.eu/).

The SDAMS is also involved in organising valuable scientific meetings. So far, it has co-organised five such meetings. The next one will be the "Discrete Biomathematics Afternoon on the Adriatic Coast", 13-14 February 2019, Koper, Slovenia (https://conferences.famnit. upr.si/event/9/).
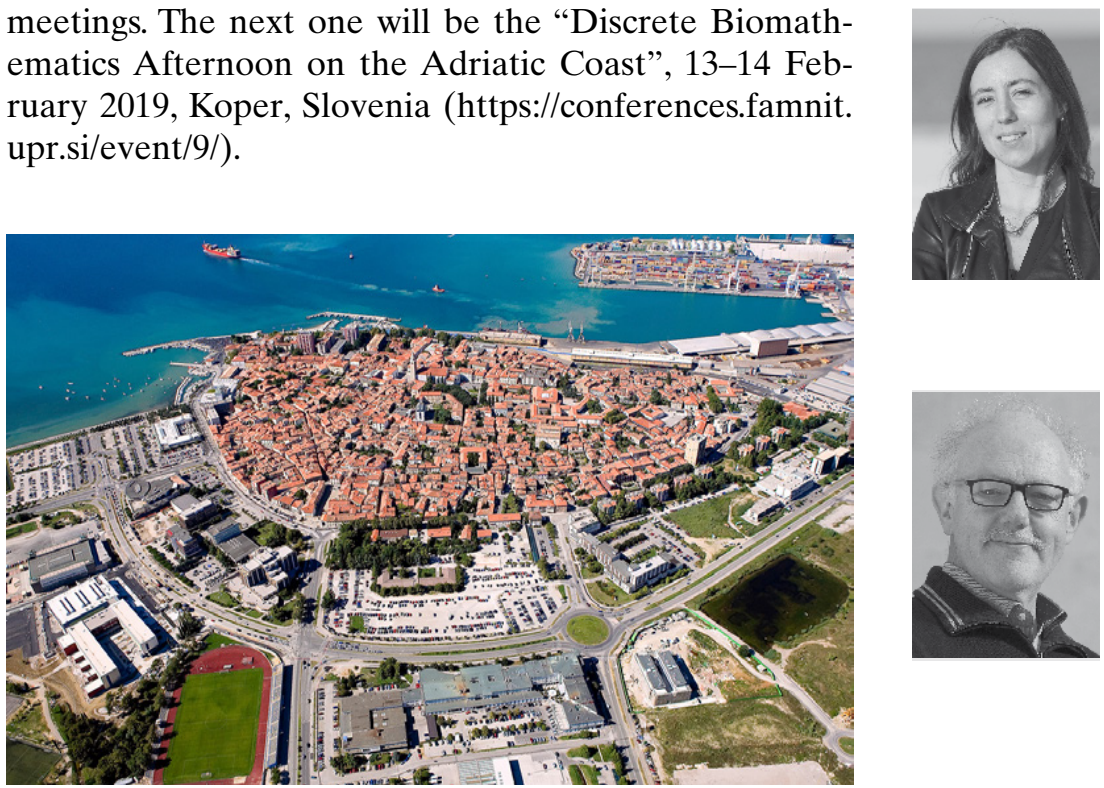

Klavdija Kutnar (Klavdija.Kutnar(at)upr. si) is a member of the executive committee of the SDAMS and the Dean of FAMNIT, the faculty of the University of Primorska, which will host 8ECM in 2020. She is the deputy chair of the organising committee of $8 E C M$. Her main research interests include algebraic graph theory.

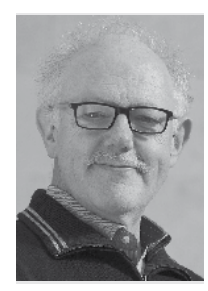

Tomaž Pisanski (Tomaz.Pisanski(at)upr. si) is the President of the SDAMS and a professor of mathematics and computer science at the University of Primorska. He is the chair of the organising committee of $8 E C M$. His research interests include various aspects of discrete mathematics. He is the co-author of a book on configurations.

Historical centre of the coastal city of Koper in Slovenia. 